\title{
Article \\ Characterization of Infant Cardiopulmonary Resuscitation Delivery with Range Sensor Feedback on Performance
}

\author{
Farah M. Alkhafaji ${ }^{1}$, Ghaidaa A. Khalid ${ }^{1, *(\mathbb{D}}$, Ali Al-Naji ${ }^{1,2, *}$, Basheer M. Hussein ${ }^{3}$ and Javaan Chahl ${ }^{2} \mathbb{C}$ \\ 1 Medical Instrumentation Techniques Engineering, Electrical Engineering Technical College, Middle Technical \\ University, Baghdad 10022, Iraq; bdc0024@mtu.edu.iq \\ 2 UniSA STEM, University of South Australia, Mawson Lakes, SA 5095, Australia; Javaan.Chahl@unisa.edu.au \\ 3 Laser and Optoelectronic Physics, College of Education for Pure Sciences, University of Kerbala, \\ Karbala 56001, Iraq; basheer.m@uokerbala.edu.iq \\ * Correspondence: ghaidaakhalid@mtu.edu.iq (G.A.K.); ali_al_naji@mtu.edu.iq (A.A.-N.)
}

Citation: Alkhafaji, F.M.; Khalid, G.A.; Al-Naji, A.; Hussein, B.M.; Chahl, J. Characterization of Infant Cardiopulmonary Resuscitation Delivery with Range Sensor Feedback on Performance. Appl. Sci. 2021, 11, 9813. https://doi.org/10.3390/ app11219813

Academic Editor: Qi-Huang Zheng

Received: 13 July 2021

Accepted: 3 October 2021

Published: 20 October 2021

Publisher's Note: MDPI stays neutral with regard to jurisdictional claims in published maps and institutional affiliations.

Copyright: () 2021 by the authors. Licensee MDPI, Basel, Switzerland. This article is an open access article distributed under the terms and conditions of the Creative Commons Attribution (CC BY) license (https:// creativecommons.org/licenses/by/ $4.0 /)$.

\begin{abstract}
Cardiac arrest (CA) in infants is an issue worldwide, which causes significant morbidity and mortality rates. Cardiopulmonary resuscitation (CPR) is a technique performed in case of CA to save victims' lives. However, CPR is often not performed effectively, even when delivered by qualified rescuers. Therefore, international guidelines have proposed applying a CPR feedback device to achieve high-quality application of CPR to enhance survival rates. Currently, no feedback device is available to guide learners through infant CPR performance in contrast to a number of adult CPR feedback devices. This study presents a real-time feedback system to improve infant CPR performance by medical staff and laypersons using a commercial CPR infant manikin. The proposed system uses an IR sensor to compare CPR performance obtained with no feedback and with a real-time feedback system. Performance was validated by analysis of the CPR parameters actually delivered against the recommended target parameters. Results show that the real-time feedback system significantly improves the quality of chest compression parameters. The two-thumb compression technique is the achievable and appropriate mechanism applied to infant subjects for delivering high-quality CPR. Under the social distancing constraints imposed by the SARS-CoV-2 pandemic, the results from the training device were sent to a CPR training center and provided each participant with CPR proficiency.
\end{abstract}

Keywords: cardiopulmonary resuscitation; SARS-CoV-2; cardiac arrest; chest compression; pandemic; chest compression; manikins; feedback; infant

\section{Introduction}

Cardiac arrest (CA) is an issue for infants around the world, which causes undesirable morbidity and mortality rates. Subjects who have CA need instant cardiopulmonary resuscitation (CPR) to save their lives. The purpose of CPR is to supply vital organs with sufficient oxygen-rich blood [1]. It is a first aid technique that allows the prevention of physiological damage while awaiting the arrival of more advanced medical intervention. It is essential to perform CPR as quickly after arrest as possible, because when cardiac arrest occurs, oxygen is no longer circulated to the brain tissues, which will result in the loss of brain function [1]. Other muscle tissues in the body are considered to be regenerative, unlike brain tissues. The CPR process involves performing alternating chest compression (CC) and artificial ventilation to physically preserve the full function of the brain of a subject who has cardiac arrest [1-3]. It is essential to increase the cardiac arrest survival rate of infant populations. Therefore, high-quality CPR is a significant factor in controlling the survival rate. When high-quality infant CPR is performed, morbidity and complications are substantially reduced. Hence, in terms of increasing the infant survival rate from cardiac arrest, significant studies and research has been undertaken by specialists working in CPR, who have presented CPR standards and recommendations. These standards and 
recommendations provide a reliable information system to supervise and document the measured quality of CC [4].

There are two types of compression techniques taught when delivering infant CPR. The Two-Finger (TF) and the Two-Thumb (TT) technique [4-6], as shown in Figure 1. The TT technique is achieved by squeezing the thorax between the two thumbs [7]. The TF technique is achieved by placing the two fingers above the lower third of the sternum and applying chest compression [6]. Thus, the only differences between the two techniques are the position of the hand. Different researchers have evaluated the effectiveness of these techniques using animal surrogates $[8,9]$ and infant CPR models $[5,10,11]$ These studies showed that performing the TT technique when delivering CPR reduces the rescuer fatigue compared to the TF technique [10]. Thus, the TT technique was advised for performing infant CPR [5-11]. The American Heart Association (AHA) and the International Liaison Committee on Resuscitation (ILCOR) have chosen the TT technique for delivering infant CPR [12]. The CPR standards are recommended by the AHA guidelines [12-14] and consider the key factors to assess the CPR quality such as: chest compression depths, chest release force, chest compression rate, and compression duty cycle.

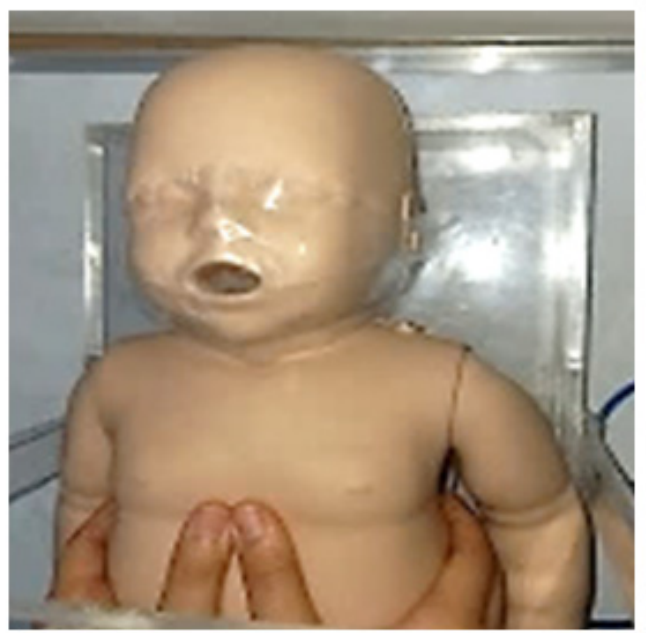

(a)

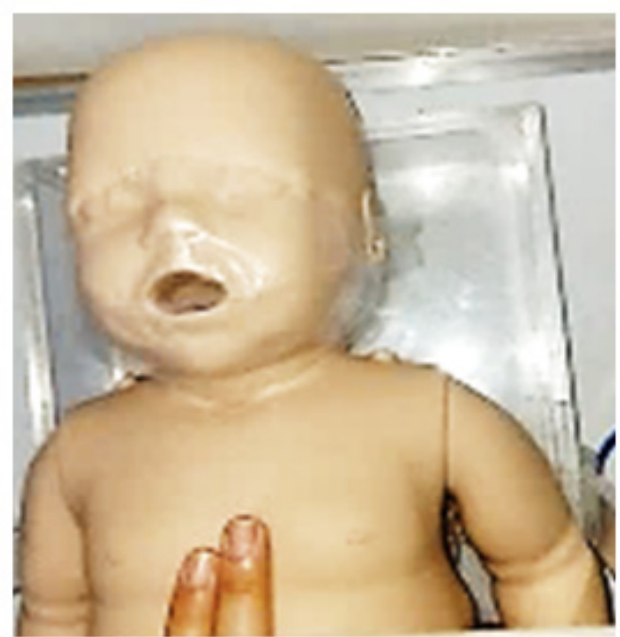

(b)

Figure 1. The Cardiopulmonary resuscitation (CPR) compressions techniques; (a) the Two-Finger (TF) method, and (b) the Two-Thumb (TT) method.

Those individuals who apply the CPR process must secure a recognized set of skills in delivering successful CPR that could save a subject's life [14,15]. Pediatric CPR differs from adult CPR because children are anatomically and physiologically different from adults [16]. In infants, cardiac arrest is not considered to be the only reason for which CPR needs to be delivered [17]. Also both hypoxia, which is "deficiency in the amount of O2 reaching tissues", and asphyxia (suffocation) are considered to be reasons for delivering of CPR [13,17]. It is challenging to identify the cause as well as the effect of infant cardiac arrest. Thus, CPR is considered to be a more difficult skill to perform on an infant or young child than adult CPR. Therefore, a training method is required to educate people about infant CPR techniques to improve the quality and rate of CPR success on infants suffering from CA $[14,18]$. For CPR to be efficient, and training to be consistent, recommended standards should be achieved. It is believed that instantaneous performance feedback could affect the performance of chest compression quality during simulated infant CPR. A study by Martin et al. [19], Kandasamy et al. [20], and Lakomek et al. [21] were conducted to investigate the feedback effect on CPR performance. Based on the outcomes from these studies, it was suggested that implementing CPR aided real-time feedback systems can improve chest compression technique. 
Currently, no feedback device is available to guide learners through infant CPR performance compared to a number of adult CPR feedback device. This study presents a real-time feedback system to improve infant CPR performance by medical staff and laypersons using commercial infant manikin. The proposed system is based on an IR sensor to analyse the CPR performance obtained with no-feedback and with a real-time feedback system. The aim of this study is to investigate and improve the CPR performance of the rescuer when delivered during simulated infant CPR.

The principal contributions of the proposed system are listed as follows: (1) Establishing background knowledge as necessary to be able to evaluate the effects of the real-time feedback system on CPR quality. (2) Propose a real-time interaction system based on an IR sensor to monitor and extract the CPR parameters and improve CPR performance in any environmental settings. (3) Propose developing a real-time feedback system to monitor and improve infant CPR performance for both medical staff and laypersons. (4) Analyze infant CPR performance with no-feedback and with a real-time feedback system and evaluate the CPR parameters delivered within the recommended target line as the main consideration. (5) Analysis of CPR performance based on the effect of using the TF and TT compression techniques and appraise the appropriate and achievable mechanism applied in infant populations for delivering high-quality infant CPR.

This paper is structured as follows: Section 2 presents the materials and methods, including participants and experimental setup. Section 3 reports the experimental results of the proposed infant CPR feedback system with comparison to the recommended guideline [12-14] of chest compression parameters. Section 4 discusses the results. Finally, Section 5 concludes the work presented in this study.

\section{Materials and Methods}

\subsection{Participants}

Simulated CPR performance was conducted across two populations, with particularly different levels of expertise: medical staff resuscitators and lay resuscitators (i.e., those who have no experience in CPR). A group of fifty persons ( 29 males and 21 females) with ages ranging between (18-56 years) was offered to participate in CPR performance on a commercial infant manikin. The participants were distributed based on their experience in CPR into two groups. Twenty-five medical staff resuscitators and twenty-five lay resuscitators enrolled for this study. Before conducting the experiments, the participants were briefed on the experimental procedure before beginning simulated CPR performance. Each participant performed four categories of infant CPR on a professional infant manikin: (1) No feedback CPR performance using the TT compression technique. (2) Feedback CPR performance using the TT compression technique. (3) No feedback CPR performance using the TF compression technique. (4) Feedback CPR performance using the TF compression technique. Individual participants' results were shown to each participant after their simulated CPR performance completion.

\subsection{Experimental Setup}

This section describes the design and development of a real-time feedback system used to improve the rescuers' performance of infant CPR working with infant populations, the full details of the technical description available in the supplementary file. The proposed system as shown in Figure 2a,b comprises (1) a commercial infant manikin (Prestan infant manikin LLC 2020, USA) was used by the participants as the infant subject.

(2) The laser displacement sensor used to measure the first CPR parameter which is chest compression depth (displacement) during the compression process. (3) An Arduino Uno Microcontroller controlled the work of the displacement sensors by sending commands to the sensors to start measuring the compression depth during TT and TF compression techniques and presenting the measures on the serial monitor of the Arduino. (4) MATLAB software (R2019a) received the readings of sensors from the Arduino in real-time and summed the reading of both sensors to calculate the actual compression depth. The other 
CPR quality parameters, compression release force, compression rate and compression duty cycle, achieved in the compression stage were calculated using the MATLAB software. (5) The performance feedback was provided by real-time program implemented using MATLAB software to both monitor and assist participants while performing CPR on the simulated infant training manikin. The CPR parameters were presented using a custom graphical user interface (GUI), see Figure 3. Applying the social distance condition imposed by the SARS-CoV-2 pandemic that made it difficult to gather all the participant in the CPR training center. Thus, a GSM chip (A9 module) controlled by the Arduino microcontroller was used to send the training results to the CPR training center to provide each participant with CPR certificate. The GUI of the proposed system contained a send option used for sending the achieved CPR performance result to CPR training center. The achieved CPR quality measures were sent from the MATLAB program to the Arduino microcontroller through a USB cable to a GSM module which transferred the data to the CPR training center, see Figure 4 . The measured data from each group were presented as mean \pm SD and median value. The statistical significance of the differences between the groups was determined by two tails, paired samples of student's test ( $\mathrm{T}$ test). The significance level for all analyses was set as $p$-value $<0.05$.

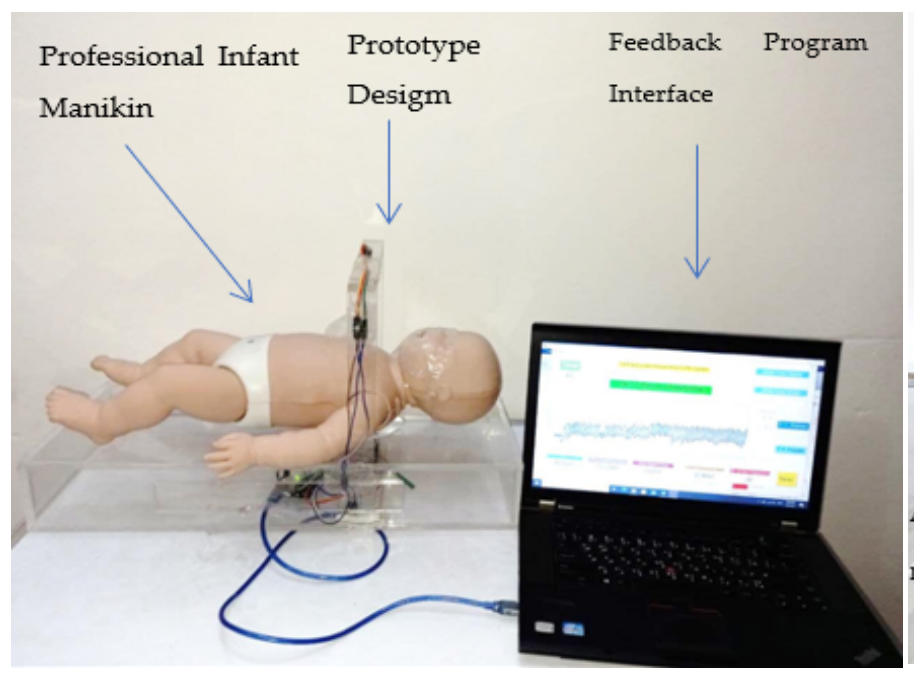

(a)

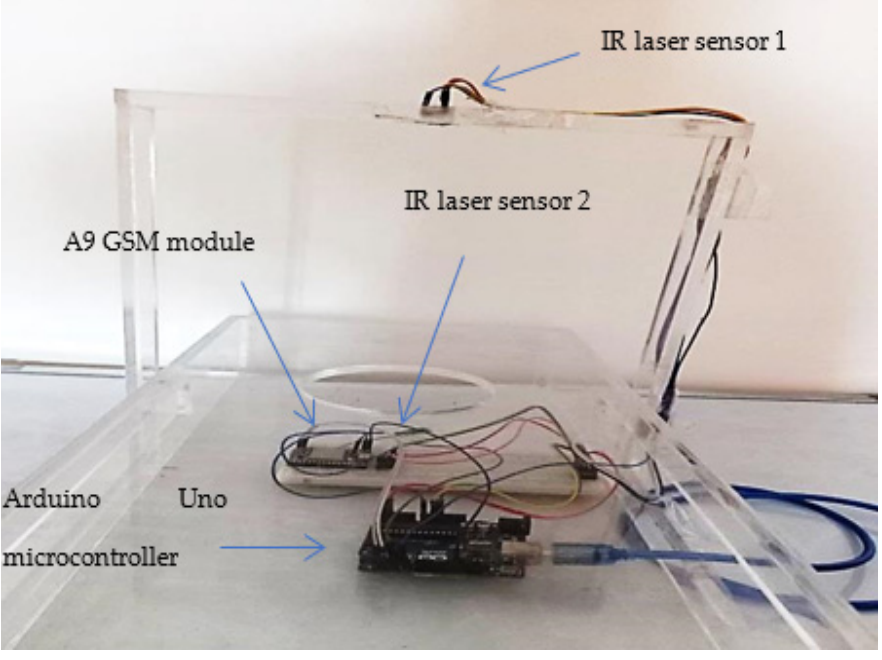

(b)

Figure 2. (a) shows the prototype of proposed system; (b) shows the electronic components of the proposed system.

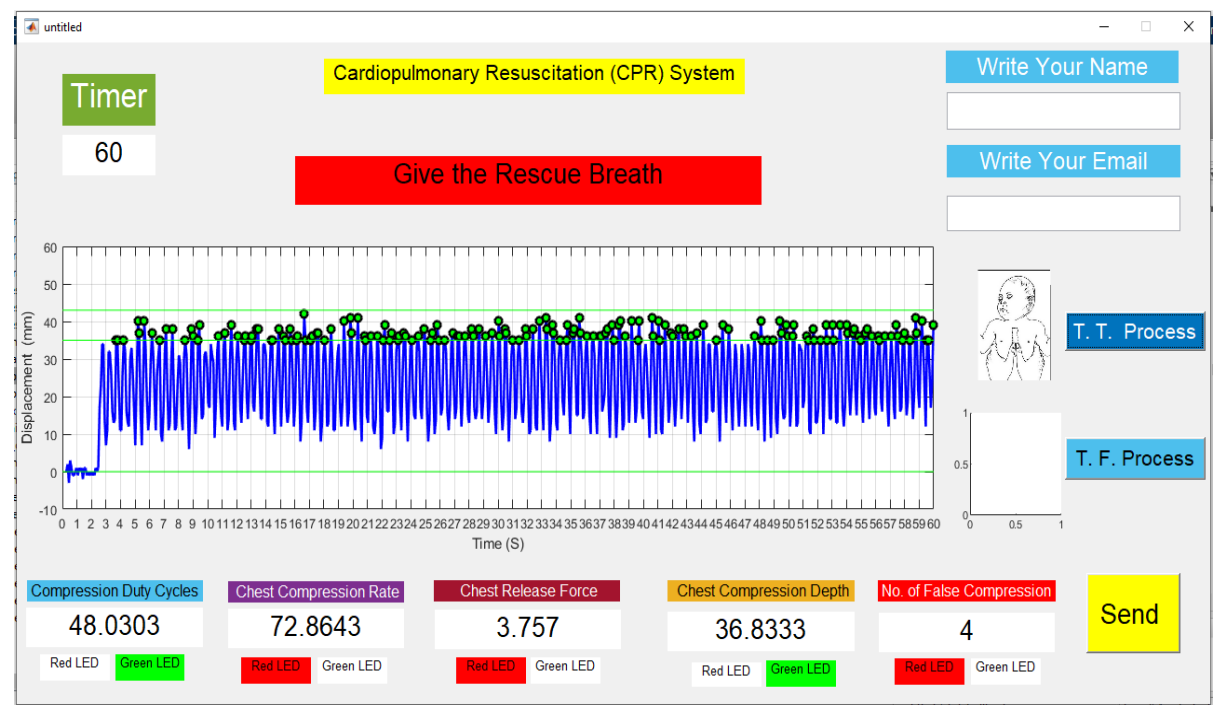

Figure 3. Shows the user interface of the proposed system. 


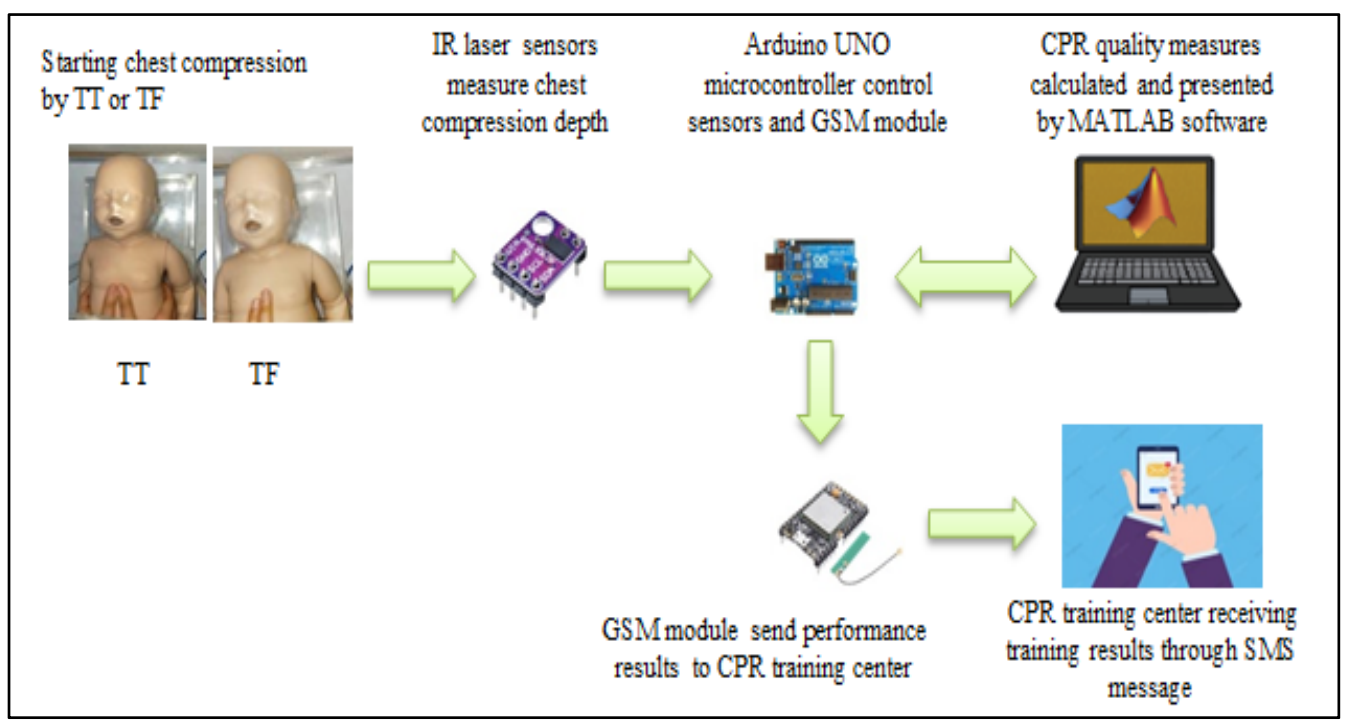

Figure 4. The overall system design of the infant CPR real-time feedback system.

\section{Results}

In this section, the results obtained are presented in two phases, the medical rescuer's CPR performance and lay rescuer's CPR performance. Measured output is presented in terms of chest compression depths, chest release force, chest compression rates and compression duty cycle. There is a supplementary material available related to this research, see Tables S1-S4 in the supplementary file.

\subsection{CPR Performance with No-Feedback and with Real-Time Feedback System}

\subsubsection{Chest Compression Depth}

The medical participants performed infant CPR using the TT compression technique see Figure 5a. The mean value of chest compression depth during no performance feedback was $32.8 \pm 2.6 \mathrm{~mm}$ compared to the chest compression depth parameter mean value of $38.2 \pm 2.7 \mathrm{~mm}$ with feedback performance. During CPR, there was an improvement in the compression depth parameter with performance feedback of approximately $16 \%$ compared to the CPR performance without feedback. The median values of the compression depth parameter with and without feedback were $33 \mathrm{~mm}$ and $38 \mathrm{~mm}$, respectively. The difference and percentage of difference between the no feedback and feedback groups were $5 \%$ and $15 \%$, respectively. There was a significant difference in the compression depth parameter when assisted with performance feedback compared to unassisted performance $(p$-value $<0.05)$.

In addition, when medically trained participants performed infant CPR using the TF compression technique, see Figure 5a. The mean value of the chest compression depth parameter without feedback was $30.2 \pm 2.07 \mathrm{~mm}$ compared to the chest compression depth parameter mean value of $36.8 \pm 1.13 \mathrm{~mm}$ with performance feedback. During CPR, there was an improvement in the compression depth parameter with feedback of approximately $22 \%$ compared to the CPR performance without feedback. The median values of the compression depth parameter with no feedback and feedback performance were $30 \mathrm{~mm}$ and $37 \mathrm{~mm}$, respectively. The difference and percentage of difference between the no feedback and feedback group were $7 \%$ and $23 \%$. There was a significant difference in the compression depth parameter with feedback-assisted performance compared to unassisted performance $(p<0.05)$. 


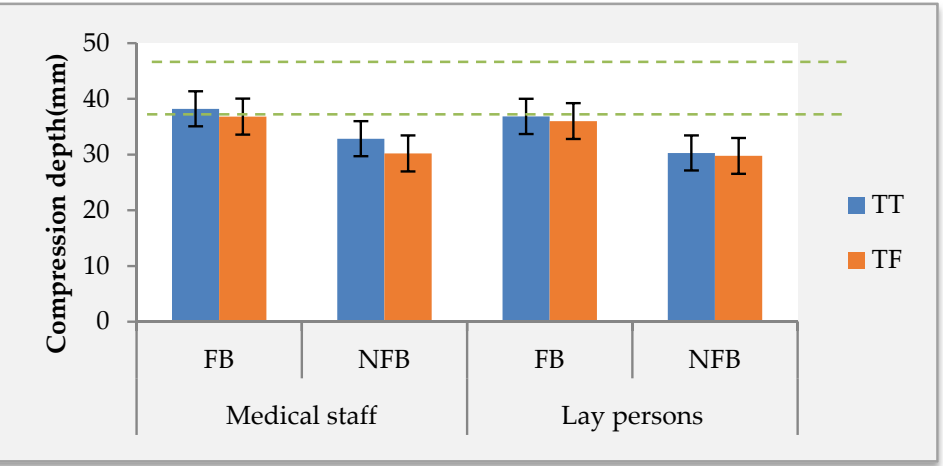

(a)

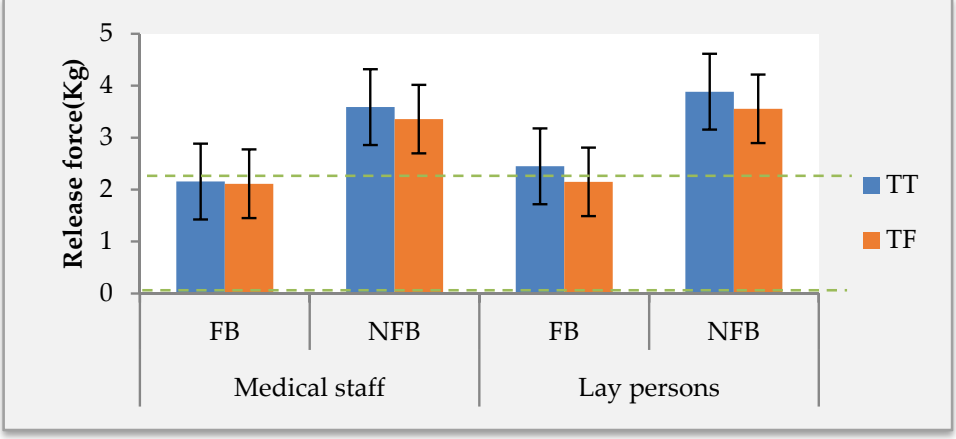

(b)

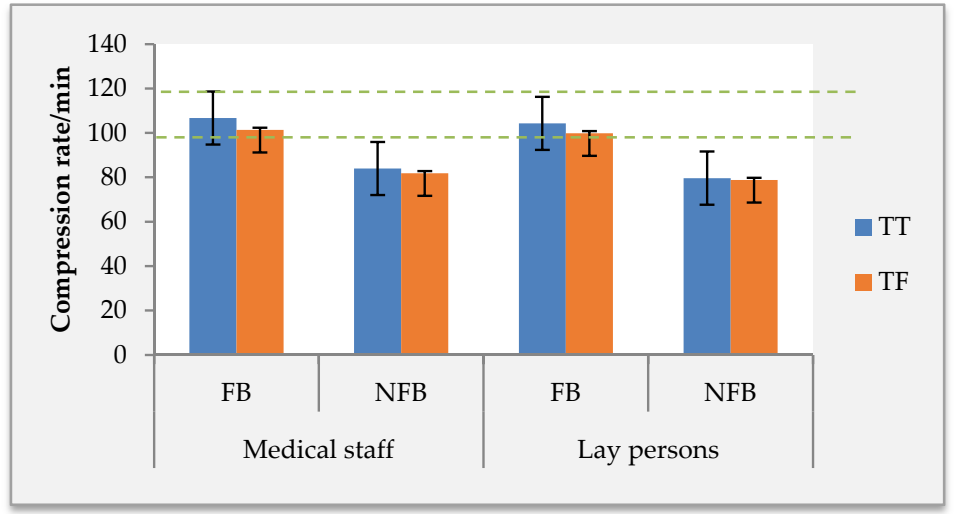

(c)

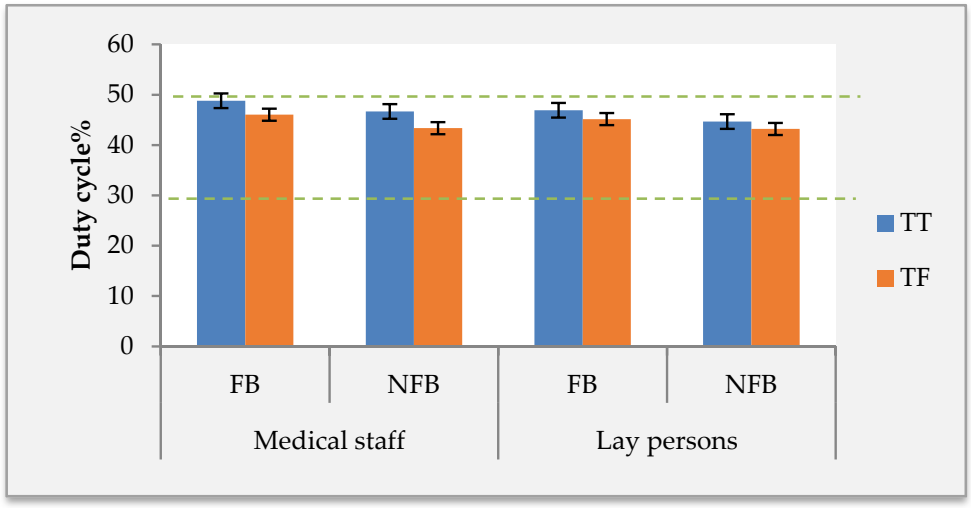

(d)

Figure 5. Mean value of the four CPR parameters achieved during no feedback and feedback CPR performance using TT and TF compression technique (a) the chest compression depth parameter; (b) the chest release force parameter; (c) the chest compression rate parameter; (d) the compression duty cycle parameter; and the dashed line represents the guideline range of each CPR parameter. 
Related to lay participants, when infant CPR was performed using the TT compression technique, see Figure 5a. The mean value of the chest compression depth parameter with no performance feedback was $30.28 \pm 3.30 \mathrm{~mm}$ compared to the chest compression depth parameter mean value of $36.84 \pm 2.16 \mathrm{~mm}$ with performance feedback. During CPR, there was an improvement in the compression depth parameter with the performance feedback of approximately $22 \%$ compared to CPR performance without feedback. The median values of the compression depth parameter during no feedback and feedback performance were $31 \mathrm{~mm}$ and $37 \mathrm{~mm}$, respectively. The difference and percentage of difference between the no feedback and feedback group were $6 \%$ and $19 \%$. There was a significant difference in the compression depth parameter with feedback-assisted performance compared to the unassisted performance ( $p$-value < 0.05).

In addition, when the lay participants performed infant CPR using the TF compression technique, see Figure 5a. The mean value of chest compression depth parameter during no feedback performance was $29.76 \pm 2.17 \mathrm{~mm}$ compared to the chest compression depth parameter mean value of $36 \pm 1.87 \mathrm{~mm}$ during feedback performance. During CPR, there was an improvement in the compression depth parameter with the feedback performance of approximately $21 \%$ compared to the CPR performance without feedback. The median values of the compression depth parameter with no feedback and feedback performances were $30 \mathrm{~mm}$ and $36 \mathrm{~mm}$, respectively. The difference and percentage of difference between the no feedback and feedback group were $6 \%$ and $20 \%$. There was a significant difference in the compression depth parameter with feedback-assisted performance compared to the unassisted performance $(p<0.05)$.

\subsubsection{Chest Release Force}

The medical participants performed infant CPR using the TT compression technique, see Figure $5 b$. The mean value of the achieved chest release force with no feedback on performance was $3.58 \pm 0.70 \mathrm{~kg}$ compared to the chest release force parameter mean value of $2.15 \pm 0.42 \mathrm{~kg}$ with feedback. The median value of the chest release force parameter with no feedback and feedback were $3.6 \mathrm{~kg}$ and $2.2 \mathrm{~kg}$, respectively. The difference and percentage of difference between the no feedback and feedback group were $1.4 \%$ and $39 \%$. During CPR, there was an improvement in the chest release force parameter with feedback approximately $40 \%$ compared to the CPR performance without feedback. There was a significant difference in the chest release force parameter during feedback-assisted performance compared to unassisted performance $(p<0.05)$.

In addition, the medical participants performed infant CPR using the TF compression technique, see Figure $5 \mathrm{~b}$. The mean value of the achieved chest release force during no feedback was $3.35 \pm 0.40 \mathrm{~kg}$ compared to the chest release force parameter mean value of $2.11 \pm 0.38 \mathrm{~kg}$ with feedback. During CPR, there was an improvement in the chest release force parameter with feedback of approximately $37 \%$ compared to the CPR performance without feedback. The median value of the chest release force parameter with no feedback and feedback on performances were $3.5 \mathrm{~kg}$ and $2.1 \mathrm{~kg}$, respectively. The difference and percentage of difference between the no feedback and feedback group were $1.4 \%$ and $40 \%$ consequently. There was a significant difference in the chest release force parameter with feedback-assisted performance compared to unassisted performance $(p<0.05)$.

The lay participants performed infant CPR using the TT compression technique, see Figure $5 \mathrm{~b}$. The mean value of the achieved chest release force using the TT compression mechanism with no performance feedback was $3.88 \pm 0.91 \mathrm{~kg}$ compared to the chest release force parameter mean value $2.44 \pm 0.60 \mathrm{~kg}$ with feedback. During CPR, there was an improvement in the chest release force parameter with feedback of approximately $37 \%$ compared to the CPR performance without feedback. The median value of the chest release force parameter during no feedback and feedback performance were $3.8 \mathrm{~kg}$ and $2.4 \mathrm{~kg}$, respectively. The difference and percentage of difference between the no feedback and feedback group were $1.4 \%$ and $37 \%$. There was a significant difference in the chest release 
force parameter with feedback-assisted performance compared to unassisted performance $(p<0.05)$.

In addition, when lay participants performed infant CPR using the TF compression technique, see Figure $5 \mathrm{~b}$. The mean value of the achieved chest release force during no performance feedback was $3.55 \pm 0.67 \mathrm{~kg}$ compared to the chest release force parameter mean value of $2.14 \pm 0.72 \mathrm{~kg}$ during feedback performance. During CPR, there was an improvement in the chest release force parameter with the feedback of approximately $40 \%$ compared to the CPR performance without feedback. The median value of the chest release force parameter during no feedback and feedback performance were $3.6 \mathrm{~kg}$ and $2.1 \mathrm{~kg}$, respectively. The difference and percentage of difference between the no feedback and feedback group were $1.5 \%$ and $42 \%$. There was a significant difference in the chest release force parameter with feedback-assisted performance compared to unassisted performance $(p<0.05)$.

\subsubsection{Compression Rate}

The medical participants performed infant CPR using the TT compression technique, see Figure 5c. The mean value of the achieved chest compression rate using the TT compression mechanism during no performance feedback was $83.96 \pm 8.87 \mathrm{cycle} / \mathrm{min}$ compared to the compression rate parameter mean value $106.68 \pm 8.43 \mathrm{cycle} / \mathrm{min}$ with feedback. The median value of the compression rate parameter with no feedback and feedback performance were $85 \mathrm{cycle} / \mathrm{min}$ and $110 \mathrm{cycle} / \mathrm{min}$, respectively. The difference and percentage of difference between the no feedback and feedback group were $25 \%$ and $29 \%$. During CPR, there was an improvement in the compression rate parameter with the feedback of approximately $27 \%$ compared to the CPR performance without feedback. There was a significant difference in the compression rate parameter with feedback-assisted performance compared to unassisted performance $(p<0.05)$.

In addition, the medical participants performed infant CPR using the TF compression technique, see Figure $5 \mathrm{c}$. The mean value of the achieved chest compression rate during no feedback was $81.84 \pm 6.65 \mathrm{cycle} / \mathrm{min}$ compared to the compression rate parameter mean value $101.36 \pm 5.16 \mathrm{cycle} / \mathrm{min}$ during feedback. During CPR, there was an improvement in the compression rate parameter with the feedback-performance of approximately $24 \%$ compared to CPR performance without feedback. The median value of the compression rate parameter during no feedback and feedback performance were $78 \mathrm{cycle} / \mathrm{min}$ and $101 \mathrm{cycle} / \mathrm{min}$, respectively. The difference and percentage of difference between the no feedback and feedback group were $23 \%$ and $29 \%$. There was a significant difference in the compression rate parameter with feedback-assisted performance compared to unassisted performance $(p<0.05)$.

Lay participants performed infant CPR using the TT compression technique, see Figure $5 c$. The mean value of the achieved chest compression rate using the TT compression mechanism during no performance feedback was $79.64 \pm 12.70 \mathrm{cycle} / \mathrm{min}$ compared to the compression rate parameter mean value $104.28 \pm 8.53$ cycle/min during performance feedback. During CPR, there was an improvement in the compression rate parameter during the feedback performance of approximately 31\% compared to CPR performance without feedback. The median value of the compression rate parameter during no feedback and feedback performance were $78 \mathrm{cycle} / \mathrm{min}$ and $108 \mathrm{cycle} / \mathrm{min}$, respectively. The difference and percentage of difference between the no feedback and feedback group were $30 \%$ and $38 \%$. There was a significant difference in the compression rate parameter with feedback-assisted performance compared to the unassisted performance $(p<0.05)$.

In addition, the lay participants performed infant CPR using the TF compression technique, see Figure 5c. The mean value of the achieved chest compression rate during no performance feedback was $78.8 \pm 6.59 \mathrm{cycle} / \mathrm{min}$ compared to the compression rate parameter mean value $99.84 \pm 10.69$ cycle/min during performance feedback. During $\mathrm{CPR}$, there was an improvement in the compression rate parameter during the feedback performance of approximately $27 \%$ compared to CPR performance without feedback. 
The median value of the compression rate parameter during no feedback and feedback performance were $78 \mathrm{cycle} / \mathrm{min}$ and $102 \mathrm{cycle} / \mathrm{min}$, respectively. The difference and percentage of difference between the no feedback and feedback group were $24 \%$ and $31 \%$. There was a significant difference in the compression rate parameter with feedback-assisted performance compared to unassisted performance $(p<0.05)$.

\subsubsection{Compression Duty Cycle}

The medical participants performed infant CPR using the TT compression technique, see Figure $5 \mathrm{~d}$. The mean value of the achieved chest compression duty cycle mechanism during no performance feedback was $46.68 \pm 3.71 \%$ compared to the compression duty cycle parameter mean value $48.8 \pm 1.95 \%$ during performance feedback. During CPR, there was an improvement in the compression duty cycle parameter during the feedback performance of approximately $5 \%$ compared to CPR performance without feedback. The median value of the compression duty cycle parameter during no feedback and feedback performance were $46 \%$ and $49 \%$, respectively. The difference and percentage of difference between the no feedback and feedback group were 3\% and 7\%. There was a significant difference in the compression duty cycle parameter with feedback-assisted performance compared to unassisted performance $(p<0.05)$.

The medical participants performed infant CPR using the TF compression technique, see Figure $5 \mathrm{~d}$. The mean value of the achieved chest compression duty cycle using the TF compression mechanism during no performance feedback was $43.36 \pm 1.89 \%$ compared to the compression duty cycle parameter mean value $46.04 \pm 2.02 \%$ with feedback. During $\mathrm{CPR}$, there was an improvement in the compression duty cycle parameter with the feedback performance of approximately $6 \%$ compared to CPR performance without feedback. The median value of the compression duty cycle parameter with no feedback and feedback performance were $43 \%$ and $46 \%$, respectively. The difference and percentage of difference between the no feedback and feedback group were 3\% and 7\%. There was a significant difference in compression duty cycle parameter with feedback-assisted performance compared to unassisted performance $(p<0.05)$. Thus, the feedback system improved the quality of the compression duty cycle parameter during CPR performance.

The lay participants performed infant CPR using the TT compression technique, see Figure $5 \mathrm{~d}$. The mean value of the achieved chest compression duty cycle during no performance feedback was $44.68 \pm 1.93 \%$ compared to the compression duty cycle parameter mean value $46.92 \pm 2.44 \%$ during feedback. During CPR, there was an improvement in the compression duty cycle parameter with the feedback of approximately $5 \%$ compared to CPR performance without feedback. The median value of the compression duty cycle parameter during no feedback and feedback performance were $45 \%$ and $47 \%$ real-time. The difference and percentage of difference between the no feedback and feedback group were $2 \%$ and $4 \%$. There was a significant difference in the compression duty cycle parameter with feedback-assisted compared to unassisted performance $(p<0.05)$.

The lay participants performed infant CPR using the TF compression technique, see Figure $5 \mathrm{~d}$. The mean value of the achieved chest compression duty cycle during no performance feedback was $43.2 \pm 2.60 \%$ compared to compression duty cycle parameter mean value $45.16 \pm 2.03 \%$ during feedback. During CPR, there was an improvement in the compression duty cycle parameter with the feedback of approximately $5 \%$ compared to CPR performance without feedback. The median value of the compression duty cycle parameter during no feedback and feedback performances were $43 \%$ and $45 \%$, respectively. The difference and percentage of the difference between the no feedback and feedback group were $2 \%$ and $5 \%$. There was a significant difference in the compression duty cycle parameter with feedback-assisted performance compared to unassisted performance $(p<0.05)$.

To assess overall CPR quality during infant CPR, the proportion of rescuers that simultaneously achieved the recommended targets of all CPR parameters was calculated for each group, medical rescuers and lay rescuers during no feedback and feedback CPR 
using the TT and TF chest compression techniques. The overall CPR quality achieved by both medical rescuers and layperson groups with feedback and without feedback from CPR using TT and TF compression techniques is shown in Figure 6.

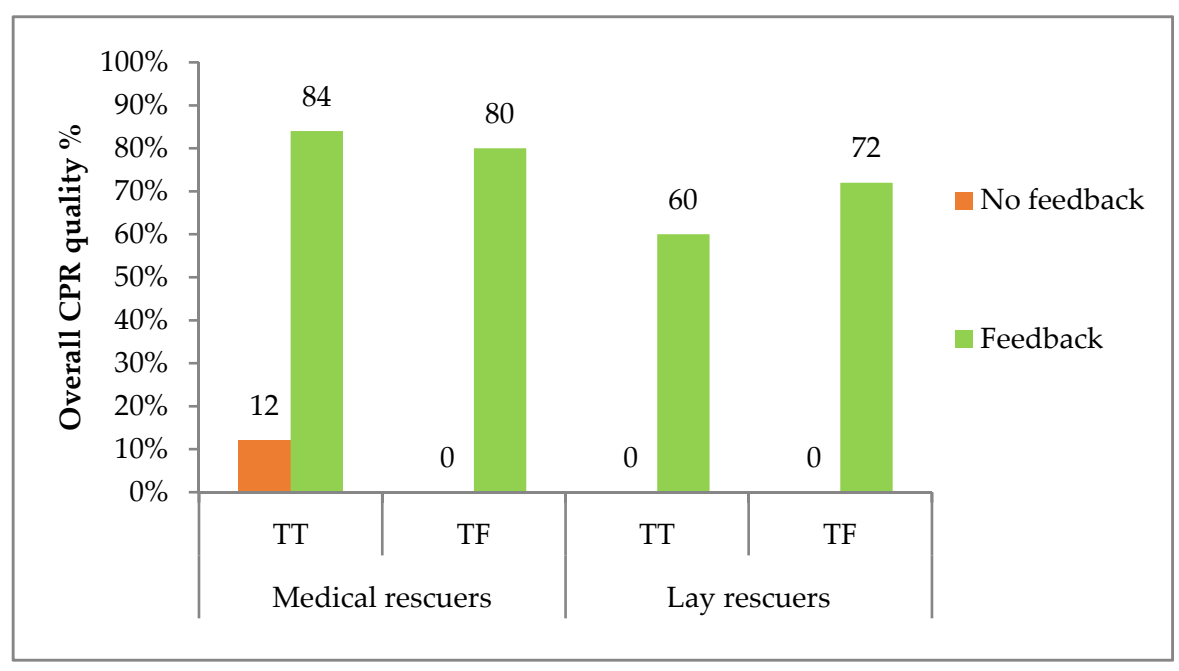

Figure 6. The overall CPR quality performance during TT and TF.

\section{Discussion}

An infant suffering from cardiac arrest (CA) generally has a high probability of immediate mortality and poor prognosis after a CA episode [1-5]. Therefore, the rescuer's quality of infant CPR should be improved during in and out of hospital cardiac arrest. Literature studies have reported that rescuers delivered poor chest compression quality during the performance of infant CPR without feedback and suggested using a realtime feedback system while performing infant CPR for monitoring and measuring the quality of infant CPR during CA [22-24]. In addition, CPR performance with a feedback assistance system required less time and fewer instructors as compared to no performance feedback [25]. Few studies have presented an optimal infant CPR feedback system in contrast to an adequate number of adult CPR systems. Some previous studies investigated the chest compression technique TT and TF during CPR performance and tried to decide the optimal chest compression technique for infant CPR performance $[5,23,26-28]$. This study aimed to develop a real-time CPR feedback system for monitoring, measuring, and improving the quality of chest compression depth, compression rate, chest release force and compression duty cycle during simulated CPR performance on an infant manikin. This section discusses the results of a series of investigations, comparing these results against literature studies and current CPR international guidelines [12-14,29]. From the results it can be seen that, in general, the assistance of the feedback CPR system has a significant effect on the quality of chest compressions during simulated infant CPR. Moreover, the internationally recommended targets $[12-14,29]$ of the four CPR parameters were achieved with the proposed feedback CPR system.

As the study was presented using two CPR methods (TT and TF), it can be seen that, in general, when feedback was not provided to the rescuer, the quality of compression depth decreased. Interestingly, when observing the feedback group, the mean compression depth achieved by rescuers was observed to be the highest. In addition, with feedback on CPR performance, the rescuers achieved deeper chest compression depth through the TT compression technique. The compression depths achieved in this study were consistent with the literature $[19,20]$

Related to chest release force, the recommended international target for chest release force is at least $0.5 \mathrm{Kg}$ and not to exceed $2.5 \mathrm{~kg}$ [29]. This study detected rescuers' failure to achieve the recommended target of chest release force without feedback infant CPR. This was comparable with previous studies that reported the role of the feedback system is 
to improve the rescuers' infant CPR performance [30,31]. However, it was observed that participants achieved the target of chest release force during simulated infant CPR using TT compression technique more easily than the TF technique, which is consistent with the outcomes reported in previous studies [26,32]. Related to the compression rate, the recommended target for compression rate was rarely achieved by rescuers who were in the group performing CPR without feedback, and most often, rescuers were not able to reach the minimum required target, which is $100 \mathrm{~min}^{-1}$. The feedback group demonstrated a significant difference across the two CPR methods. However, the rescuers achieved the recommended target compression rate during simulated infant CPR more easily when using the TT compression technique than the TF technique. This was consistent with the compression rate parameter reported by $[6,32,33]$. The compression duty cycle proved difficult to maintain, particularly when feedback was not provided. This could be due to difficulty in understanding how to maintain the duty cycle within the required limits, or due to a loss of concentration, perhaps as a result of focusing on the compression rates and depths. A relatively significant improvement occurred with the group provided with feedback, since overall, the international recommended targets were achieved. More rescuers reached the recommended guideline of duty cycle through chest compression using the TT technique than the TF technique. This performance was in agreement with the reported studies in [27,34]. The study also investigated the effect of real-time performance feedback during simulated infant chest compressions in accordance with the method described by $[19,35]$. The researcher showed that feedback produced an overall improvement in the quality of chest compression, significantly improving the quality of chest compression depths, chest compression rates and compression duty cycles. Although, that study focused only on the TT technique. The results of this current study have produced similar outcomes to [19,35]. Comparing the current study with [19,35], it can be seen that the group of rescuers with feedback had significantly increased performance quality for four of the quality measurements. The rescuer group without feedback demonstrated a deterioration in performance quality; most participants failed to achieve all four (compression depth, compression rates, release force and duty cycle) quality targets.

Overall, the study analysis related to the four quality parameters of CPR performance on an infant manikin, the chest compression depth, chest release force, compression rate and compression duty cycle has demonstrated that the TT compression technique is better to achieve the recommended target of CPR quality parameters. Therefore, the TT compression technique was advised for infant CPR performance. The proposed system in this study could be used to assist rescuers in improving the quality of CPR performance.

The limitations of the study represented by (1) the SARS-CoV-2 pandemic prevent rescuers from achieving rescue breath parameters. (2) Ending of the result did not include the sampled signal since the GSM could not send more than 26 characters in one message.

\section{Conclusions}

Currently, infant CPR training is usually performed without a feedback system, which may cause poor CPR performance delivered by rescuers in comparison to the adequate numbers of adult CPR feedback systems. This study presents a real-time feedback system to assist rescuers to achieve quality measures of CPR parameters. This study investigated the CPR performance delivered by medical rescuers and lay rescuers on a commercial infant manikin without an assisting feedback system and with a real-time assisting feedback system. The results showed some ineffective CPR performance during no feedback CPR performance against improved CPR quality measures during CPR performance with the assisting feedback system. Furthermore, this study showed that the TT compression technique is preferable to the TF compression technique when performing infant CPR. Further, the proposed system's capability of transmitting CPR performance is very valuable because it supports remote CPR training under the social distancing conditions of the SARSCoV-2 pandemic. 
Supplementary Materials: The following are available online at https: / www.mdpi.com/article / 10.3390/app11219813/s1, Table S1: The result of medical rescuers group during feedback and no feedback CPR performance of the CPR quality parameters by TT compression technique.; Table S2: The result of medical rescuers group during feedback and no feedback CPR performance of the CPR quality parameters by TF compression technique.; Table S3: The result of lay rescuers group during feedback and no feedback CPR performance of the CPR quality parameters by TT compression method.; Table S4: The result of lay rescuers group during feedback and no feedback CPR performance of the CPR quality parameters by TF compression method.

Author Contributions: Conceptualization, A.A.-N. and G.A.K.; methodology, G.A.K. and F.M.A.; software, G.A.K. and B.M.H.; validation, F.M.A. and G.A.K.; formal analysis, F.M.A. and G.A.K.; investigation, F.M.A., G.A.K. and A.A.-N.; resources, F.M.A., G.A.K. and B.M.H.; data curation, F.M.A., G.A.K. and A.A.-N.; writing—original draft preparation, F.M.A. and G.A.K.; project funding, J.C.; writing-review and editing, F.M.A., G.A.K., A.A.-N. and J.C.; visualization, F.M.A., G.A.K. and A.A.-N.; project administration, G.A.K., A.A.-N. and J.C. All authors have read and agreed to the published version of the manuscript.

Funding: The research has received no funding.

Institutional Review Board Statement: The study followed the guidelines of the Declaration of Helsinki, and approved by the Ministry of Health and Environment, Training and Human Development Centre, Iraq (Protocol number: 84/21).

Informed Consent Statement: Informed consent was obtained from all subjects involved in the study to publish this paper.

Data Availability Statement: Not applicable.

Conflicts of Interest: The authors declare no conflict of interest.

\section{References}

1. Mendler, M.R.; Weber, C.; Hassan, M.A.; Huang, L.; Waitz, M.; Mayer, B.; Hummler, H.D. Effect of Different Respiratory Modes on Return of Spontaneous Circulation in a Newborn Piglet Model of Hypoxic Cardiac Arrest. Neonatology 2015, 109, 22-30. [CrossRef] [PubMed]

2. Solevåg, A.L.; Schmölzer, G.M.; O’Reilly, M.; Lu, M.; Lee, T.-F.; Hornberger, L.K.; Nakstad, B.; Cheung, P.-Y. Myocardial perfusion and oxidative stress after $21 \%$ vs. $100 \%$ oxygen ventilation and uninterrupted chest compressions in severely asphyxiated piglets. Resuscitation 2016, 106, 7-13. [CrossRef]

3. Cunningham, L.M.; Mattu, A.; O'Connor, R.E.; Brady, W.J. Cardiopulmonary resuscitation for cardiac arrest: The importance of uninterrupted chest compressions in cardiac arrest resuscitation. Am. J. Emerg. Med. 2012, 30, 1630-1638. [CrossRef] [PubMed]

4. Nolan, J. High-quality cardiopulmonary resuscitation. Curr. Opin. Crit. Care 2014, 20, 227-233. [CrossRef] [PubMed]

5. Dorfsman, M.L.; Menegazzi, J.J.; Wadas, R.J.; Auble, T.E. Two-thumb vs. two-finger chest compression in an infant model of prolonged cardiopulmonary resuscitation. Acad. Emerg. Med. 2000, 7, 1077-1082. [CrossRef]

6. Tsou, J.-Y.; Kao, C.-L.; Chang, C.-J.; Tu, Y.-F.; Su, F.-C.; Chi, C.-H. Biomechanics of two-thumb versus two-finger chest compression for cardiopulmonary resuscitation in an infant manikin model. Eur. J. Emerg. Med. 2020, 27, 132-136. [CrossRef]

7. Alkhafaji, F.M.; Khalid, G.A.; Al-Naji, A. Application of Cardiopulmonary Resuscitation Mechanism in Infant Population: A Short Review. IOP Conf. Ser. Mater. Sci. Eng. 2021, 1105, 012077. [CrossRef]

8. Menegazzi, J.J.; Auble, T.E.; Nicklas, K.A.; Hosack, G.M.; Rack, L.; Goode, J.S. Two-thumb versus two-finger chest compression during CPR in a swine infant model of cardiac arrest. Ann. Emerg. Med. 1993, 22, 240-243. [CrossRef]

9. Houri, P.K.; Frank, L.R.; Menegazzi, J.J.; Taylor, R. A randomized, controlled trial of two-thumb vs two-finger chest compression in a swine infant model of cardiac arrest [see comment]. Prehosp. Emerg. Care 1997, 1, 65-67. [CrossRef]

10. Haque, I.U.; Udassi, J.P.; Udassi, S.; Theriaque, D.W.; Shuster, J.J.; Zaritsky, A.L. Chest compression quality and rescuer fatigue with increased compression to ventilation ratio during single rescuer pediatric CPR. Resuscitation 2008, 79, 82-89. [CrossRef]

11. Whitelaw, C.C.; Slywka, B.; Goldsmith, L. Comparison of a two-finger versus two-thumb method for chest compressions by healthcare providers in an infant mechanical model. Resuscitation 2000, 43, 213-216. [CrossRef]

12. Merchant, R.M.; Topjian, A.A.; Panchal, A.R.; Cheng, A.; Aziz, K.; Berg, K.M.; Lavonas, E.J.; Magid, D.J. Part 1: Executive Summary: 2020 American Heart Association Guidelines for Cardiopulmonary Resuscitation and Emergency Cardiovascular Care. Circulation 2020, 142, S337-S357. [CrossRef] [PubMed]

13. De Caen, A.R.; Kleinman, M.E.; Chameides, L.; Atkins, D.L.; Berg, R.A.; Berg, M.D.; Bhanji, F.; Biarent, D.; Bingham, R.; Coovadia, A.H. Part 10: Paediatric basic and advanced life support: International consensus on cardiopulmonary resuscitation and emergency cardiovascular care science with treatment recommendations. Resuscitation 2010, 81, 213-259. [CrossRef] [PubMed] 
14. Atkins, D.L.; Berger, S.; Duff, J.P.; Gonzales, J.C.; Hunt, E.A.; Joyner, B.L.; Maeney, P.A.; Niles, D.E.; Samson, R.A.; Schexnayder, S.M. Part 11: Pediatric basic life support and cardiopulmonary resuscitation quality: 2015 American Heart Association guidelines update for cardiopulmonary resuscitation and emergency cardiovascular care. Circulation 2015, 132, S519-S525. [CrossRef] [PubMed]

15. Biarent, D.; Bingham, R.; Eich, C.; López-Herce, J.; Maconochie, I.; Rodríguez-Núnez, A.; Rajka, T.; Zideman, D. European Resuscitation Council Guidelines for Resuscitation 2010 Section 6. Paediatric life support. Resuscitation 2010, 81, $1364-1388$. [CrossRef] [PubMed]

16. Atkins, D.L.; Berger, S. Improving Outcomes from Out-of-Hospital Cardiac Arrest in Young Children and Adolescents. Pediatr. Cardiol. 2011, 33, 474-483. [CrossRef]

17. Bardai, A.; Berdowski, J.; van der Werf, C.; Blom, M.T.; Ceelen, M.; van Langen, I.M.; Tijssen, J.G.; Wilde, A.A.; Koster, R.W.; Tan, H.L. Incidence, Causes, and Outcomes of Out-of-Hospital Cardiac Arrest in Children A Comprehensive, Prospective, Population-Based Study in the Netherlands. J. Am. Coll. Cardiol. 2011, 57, 1822-1828. [CrossRef]

18. Udassi, J.P.; Udassi, S.; Theriaque, D.W.; Shuster, J.J.; Zaritsky, A.L.; Haque, I.U. Effect of alternative chest compression techniques in infant and child on rescuer performance. Pediatr. Crit. Care Med. 2009, 10, 328-333. [CrossRef]

19. Martin, P.; Theobald, P.; Kemp, A.; Maguire, S.; Maconochie, I.; Jones, M. Real-time feedback can improve infant manikin cardiopulmonary resuscitation by up to 79\%-A randomised controlled trial. Resuscitation 2013, 84, 1125-1130. [CrossRef]

20. Kandasamy, J.; Theobald, P.S.; Maconochie, I.K.; Jones, M.D. Can real-time feedback improve the simulated infant cardiopulmonary resuscitation performance of basic life support and lay rescuers? Arch. Dis. Child. 2019, 104, 793-801. [CrossRef]

21. Lakomek, F.; Lukas, R.-P.; Brinkrolf, P.; Mennewisch, A.; Steinsiek, N.; Gutendorf, P.; Sudowe, H.; Heller, M.; Kwiecien, R.; Zarbock, A.; et al. Erratum: Real-time feedback improves chest compression quality in out-of-hospital cardiac arrest: A prospective cohort study. Plos One 2020, 15, e229431. [CrossRef]

22. Abella, B.S.; Edelson, D.P.; Kim, S.; Retzer, E.; Myklebust, H.; Barry, A.M.; O’Hearn, N.; Hoek, T.L.V.; Becker, L.B. CPR quality improvement during in-hospital cardiac arrest using a real-time audiovisual feedback system. Resuscitation 2007, 73, 54-61. [CrossRef]

23. Lin, Y.; Cheng, A.; Grant, V.J.; Currie, G.R.; Hecker, K.G. Improving CPR quality with distributed practice and real-time feedback in pediatric healthcare providers-A randomized controlled trial. Resuscitation 2018, 130, 6-12. [CrossRef] [PubMed]

24. Krasteva, V.; Jekova, I.; Didon, J.-P. An audiovisual feedback device for compression depth, rate and complete chest recoil can improve the CPR performance of lay persons during self-training on a manikin. Physiol. Meas. 2011, 32, 687-699. [CrossRef] [PubMed]

25. Gregson, R.K.; Cole, T.; Skellett, S.; Bagkeris, E.; Welsby, D.; Peters, M. Randomised crossover trial of rate feedback and force during chest compressions for paediatric cardiopulmonary resuscitation. Arch. Dis. Child. 2016, 102, 403-409. [CrossRef]

26. Jiang, J.; Zou, Y.; Shi, W.; Zhu, Y.; Tao, R.; Jiang, Y.; Lu, Y.; Tong, J. Two-thumb-encircling hands technique is more advisable than 2-finger technique when lone rescuer performs cardiopulmonary resuscitation on infant manikin. Am. J. Emerg. Med. 2015, 33, 531-534. [CrossRef] [PubMed]

27. Christman, C.; Hemway, R.J.; Wyckoff, M.H.; Perlman, J.M. The two-thumb is superior to the two-finger method for administering chest compressions in a manikin model of neonatal resuscitation. Arch. Dis. Child. Fetal Neonatal Ed. 2010, 96, F99-F101. [CrossRef] [PubMed]

28. Huynh, T.K.; Hemway, R.J.; Perlman, J.M. The Two-Thumb Technique Using an Elevated Surface is Preferable for Teaching Infant Cardiopulmonary Resuscitation. J. Pediatr. 2012, 161, 658-661. [CrossRef]

29. Kleinman, M.E.; De Caen, A.R.; Chameides, L.; Atkins, D.L.; Berg, R.A.; Berg, M.C.; Bhanji, F.; Biarent, D.; Bingham, R.; Coovadia, A.H. Part 10: Pediatric basic and advanced life support: 2010 International Consensus on Cardiopulmonary Resuscitation and Emergency Cardiovascular Care Science with Treatment Recommendations. Circulation 2010, 122, 971093. [CrossRef]

30. Kitamura, T.; Iwami, T.; Kawamura, T.; Nagao, K.; Tanaka, H.; Nadkarni, V.M.; Berg, R.A.; Hiraide, A. Conventional and chestcompression- only cardiopulmonary resuscitation by bystanders for children who have out-of-hospital cardiac arrests: A prospective, nationwide, populationbased cohort study. Lancet 2010, 375, 1347-1354. [CrossRef]

31. López-Herce, J.; García, C.; Dominguez-Sampedro, P.; Rodriguez-Nunez, A.; Carrillo, A.; Calvo, C.; Delgado, M.A.; Spanish Study Group of Cardiopulmonary Arrest in Children. Outcome of Out-of-Hospital Cardiorespiratory Arrest in Children. Pediatr. Emerg. Care 2005, 21, 807-815. [CrossRef]

32. Udassi, S.; Udassi, J.P.; Lamb, M.A.; Theriaque, D.W.; Shuster, J.J.; Zaritsky, A.L.; Haque, I.U. Two-thumb technique is superior to two-finger technique during lone rescuer infant manikin CPR. Resuscitation 2010, 81, 712-717. [CrossRef]

33. Reynolds, C.; Cox, J.; Livingstone, V.; Dempsey, E.M. Rescuer Exertion and Fatigue Using Two-Thumb vs. Two-Finger Method During Simulated Neonatal Cardiopulmonary Resuscitation. Front. Pediatr. 2020, 8, 133. [CrossRef]

34. Millin, M.G.; Bogumil, D.; Fishe, J.N.; Burke, R.V. Comparing the two-finger versus two-thumb technique for single person infant CPR: A systematic review and meta-analysis. Resuscitation 2020, 148, 161-172. [CrossRef]

35. Martin, P.S.; Kemp, A.M.; Theobald, P.; Maguire, S.A.; Jones, M.D. Do chest compressions during simulated infant CPR comply with international recommendations? Arch. Dis. Child. 2012, 98, 576-581. [CrossRef] [PubMed] 